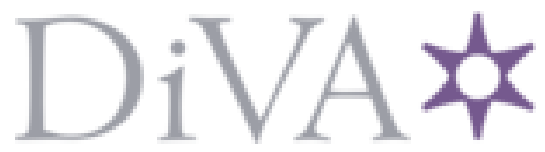

http://www.diva-portal.org

This is the published version of a paper presented at IEEE Conference on Cybernetics and Intelligent Systems.

Citation for the original published paper:

Fleyeh, H. (2004)

COLOR DETECTION AND SEGMENTATION FOR ROAD AND TRAFFIC SIGNS

In: IEEE (ed.), CIS-RAM 2004 (pp. 809-814). Singapore

N.B. When citing this work, cite the original published paper.

Permanent link to this version:

http://urn.kb.se/resolve?urn=urn:nbn:se:du-30870 


\title{
COLOR DETECTION AND SEGMENTATION FOR ROAD AND TRAFFIC SIGNS
}

\author{
Hasan Fleyeh \\ hfl@du.se \\ Department of Computer Engineering, Dalarna University, Sweden \\ Guest Researcher, Transportation Research Institute, Napier University, Scotland
}

\begin{abstract}
This paper aims to present three new methods for color detection and segmentation of road signs. The images are taken by a digital camera mounted in a car. The RGB images are converted into IHLS color space, and new methods are applied to extract the colors of the road signs under consideration. The methods are tested on hundreds of outdoor images in different light conditions, and they show high robustness. This project is part of the research taking place in Dalarna University / Sweden in the field of the ITS.
\end{abstract}

Keywords: Color segmentation, color detection, road signs, outdoor images.

\section{Introduction}

Road signs and traffic signals define a visual language that can be interpreted by drivers. They represent the current traffic situation on the road, show danger and difficulties around the drivers, give them warnings, and help them with their navigation by providing useful information that makes driving safe and convenient $[1,2]$.

The human visual perception abilities depend on the individual's physical and mental conditions. In certain circumstances, these abilities can be affected by many factors such as fatigue, and observatory skills. Giving this information in a good time to drivers can prevent accidents, save lives, increase driving performance, and reduce the pollution caused by vehicles [3-5].

Colors represent an important part of the information provided to the driver to ensure the objectives of the road sign. Therefore, road signs and their colors are selected to be different from the nature or from the surrounding in order to be distinguishable. Detection of these signs in outdoor images from a moving vehicle will help the driver to take the right decision in good time, which means fewer accidents, less pollution, and better safety.

About $62 \%$ of the reviewed literature used colors as the basic cue for road sign detection, the remaining used shape. RGB images are converted by Vitabile and Sorbello [6] into HSV color space which is divided into a number of subspaces (regions). The $\mathrm{S}$ and $\mathrm{V}$ components are used to find in which region the hue is located. Paclik et al. [7] segmented the color images by using HSV color space. Colors like red, blue, green, and yellow were segmented by $\mathrm{H}$ component and a certain threshold. Vitabile et al. [8] proposed a dynamic, optimized HSV sub-space, according to the $s$ and $v$ values of the processed images. Color segmentation was achieved by Vitabile et. al. [9, 10] by using priori knowledge about color signs in the HSV system. de la Escalera et al. [11] built a color classifier based on two look-up tables derived from hue and saturation of an HSI color space. Fang et al. [2] developed a road sign detection and tracking system in which the color images from a video camera are converted into HSI system. Color features are extracted from the hue by using a twolayer neural network.

The remaining of the paper is organized as follows. Section 2 describes the properties of the road signs. Section 3 shows the difficulties behind working in the outdoor scenes, and the effect of different factors on the perceived images. Section 4 describes the improved HLS color space, and section 5 describes how the color varies in the outdoor images and the parameters affecting this. In section 6 , there is a description of the properties of the hue and how it changes due to light variations. Section 7 shows the segmentation methods, and section 8 shows the result and future research.

\section{Road and Traffic Signs}

Road and traffic signs have been designed using special shapes and colors, very different from the natural environment, which make them easily recognizable by drivers [12]. These may be principally distinguishable from the natural and/or man-made backgrounds [13]. They are designed, manufactured and installed according to stringent regulations [6]. They are designed in fixed 2-D shapes like triangles, circles, octagons, or rectangles $[14,15]$. The colors are regulated to the sign category $(\mathrm{red}=$ stop, yellow $=$ danger) $[16]$. 
The information on the sign has one color and the rest of the sign has another color. The tint of the paint which covers the sign should correspond to a specific wavelength in the visible spectrum $[6,10]$. The signs are located in well-defined locations with respect to the road, so that the driver can, more or less, expect the location of these signs [16]. They may contain a pictogram, a string of characters or both [10]. The road signs are characterized by using fixed text fonts, and character heights. They can appear in different conditions, including partly occulted, distorted, damaged and clustered in a group of more than one sign $[10,14]$.

\section{Difficulties}

Due to the complex environment of the roads and the scenes around them, the detection and recognition of road and traffic signs may face many difficulties such as:

○ The color of the sign fades with time as a result of long exposure to sun light, and the reaction of the paint with the air $[1,5]$.

- The visibility is affected by weather conditions such as fog, rain, clouds and snow [1]. Other parameters like local light variations (the direction of the light, the strength of the light depending on the time of the day and the season), and the shadows generated by other objects $[9,10,17]$ can also affect visibility.

- The color information is very sensitive to the variations of the light conditions such as shadows, clouds, and the sun. [1, 5, 8]. It can be affected by the illuminant color (daylight), illumination geometry, and viewing geometry [18].

- The presence of objects similar in color and/or shapes to the road signs in the scene under consideration, like buildings, or vehicles [5, 17].

- Signs may be found disoriented, damaged or occulted.

o If the image is acquired from a moving car, then it is often suffers from motion blur and car vibration [19].

- The presence of obstacles in the scene, like trees, buildings, vehicles and pedestrians [8, 17].

- Another drawback is the absence of a standard database for evaluation of the existing classification methods [7].

\section{The Improved HLS Color Space}

Hanbury and Serra [20] introduced an improved version of HLS color space which was later called IHLS. This color space is very similar to the other color spaces, but it avoids the inconveniences of the other color spaces designed for computer graphics rather than image processing. The color space provides independence between chromatic and achromatic components [21]. The conversion from the RGB to this color space is calculated as follows:

$$
\begin{aligned}
& H=\theta \quad \text { if } B \leq G \\
& H=360-\theta \quad \text { if } B>G
\end{aligned}
$$

where:

$$
\theta=\cos ^{-1}\left\{\frac{\left[R-\frac{G}{2}-\frac{B}{2}\right]}{\sqrt{R^{2}+G^{2}+B^{2}-R G-R B-G B}}\right\}
$$

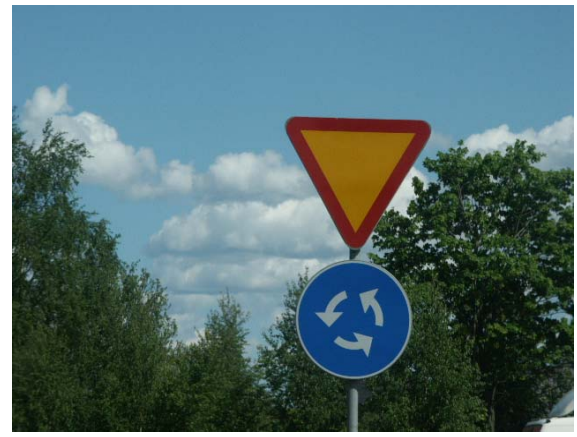

(A)

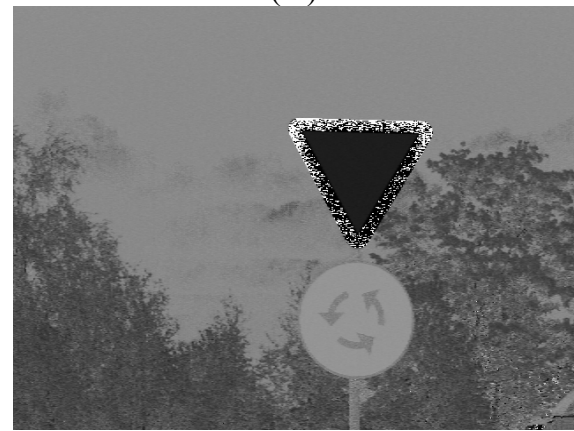

(B)

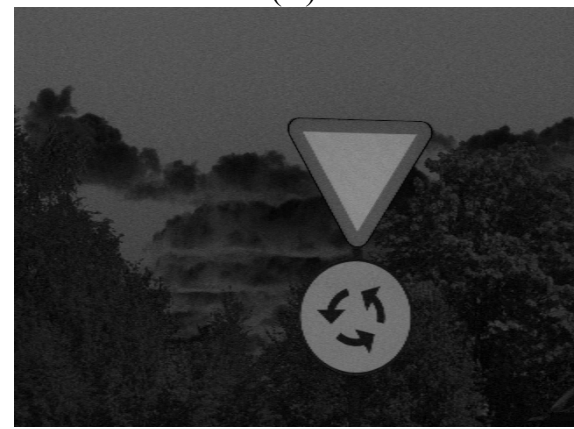

(C)

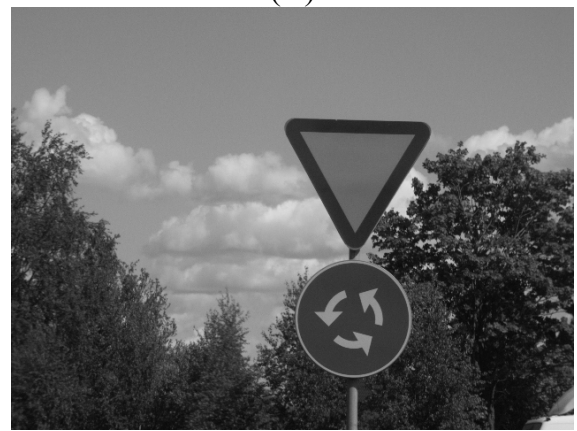

(D)

Figure 1 (A) Original image, (B) Normalized Hue, (C) Normalized Saturation, (D) Luminance 
The other two parameters are calculated as follows:

$S=\max (R, G, B)-\min (R, G, B)$

$L=0.212 R+0.715 G+0.072 B$

Figure 1 shows the hue, saturation, and luminance of this color space.

\section{Color Variations in the Outdoor Images}

One of the most difficult problems in using the colors in the outdoor images is the chromatic variation of daylight. As a result of this chromatic variation, the apparent color of the object varies as daylight changes.

The irradiance of any object in a color image depends on three parameters:

The color of the incident light, its intensity and the position of the light source: The color of the daylight varies along the characteristic curve in the CIE model. It is given by the following equation:

$y=2.87 x-3.0 x^{2}-0.275$ for $0.25 \leq x \leq 0.38$.

According to this equation, the variation of the daylight's color is a single variable change called the temperature of the daylight, which is independent of the intensity.

The reflectance properties of the object: The reflectance of an object $s(\lambda)$ is a function of the wavelength $\lambda$ of the incident light. It is given by: $s(\lambda)=e(\lambda) \phi(\lambda)$. Where $e(\lambda)$ is the intensity of the light at wavelength $\lambda$, and $\phi(\lambda)$ is the object's albedo function giving the percent of the light reflected at each wavelength. This model did not take into consideration the extended light sources, inter-reflectance effects, shadowing or specularities, but it is the best available working model of color reflectance.

The camera properties: Given the radiance of an object $L(\lambda)$, the observed intensities depend on the lens diameter $d$, the focal length $f$ of the camera, and the image position of the object measured as angle $a$ off the optical axis. This is given by the standard irradiance equation

$E(\lambda)=L(\lambda) \cdot(\pi / 4)(d / f)^{2} \cos (4 a)$. According to this equation, the radiance $L(\lambda)$ is multiplied by a constant function of the camera parameters. This means that it will not affect the observed color of the object. Assuming that the chromatic aberration of the camera's lens is negligible, only the density of the observed light will be affected.

As a result, the color of the light reflected by an object located outdoors is a function of the temperature of the daylight and the object's albedo, and the observed irradiance is the reflected light surface scaled by the irradiance equation $[18,22]$.

\section{Hue Properties and Adrift due to Illumination Changes}

In some color spaces, the hue plays a central role in the color detection. This is because it is invariant to the variations in light conditions as it is multiplicative/scale invariant, additive/shift invariant, and it is invariant under saturation changes. But the hue coordinate is unstable, and small changes in the RGB can cause strong variation in hue [16], and it suffers from three problems. Firstly, when the intensity is very low or very high, the hue is meaningless. Secondly, when the saturation is very low, the hue is meaningless. Thirdly, when the saturation is less than a certain threshold, the hue becomes unstable.

Vitabile et al. [10] defined three different areas in the HSV color space: The achromatic area: characterized by $s \leq 0.25$ or $v \leq 0.2$ or $v \geq 0.9$. The unstable chromatic area: characterized by $0.25 \leq s \leq 0.5$ and $0.2 \leq v \leq 0.9$. The chromatic area: characterized by $s \geq 0.5$ and $0.2 \leq v \leq 0.9$.

In order to obtain robustness against changes in external light conditions, these areas should be taken into consideration in any color segmentation system.

\section{Color image segmentation}

\subsection{Method 1:}

Color segmentation is carried out by converting the RGB image into the IHLS system. Three images are generated as shown in figure 1. The global mean of the luminance image is calculated by:

mean $=\frac{1}{m n} \sum_{i=0}^{m-1} \sum_{j=0}^{n-1} L(i, j)$

Nmean $=$ mean $/ 256$

where $m$ and $n$ are the image dimensions, $L(i, j)$ is the luminance of the current pixel, and Nmean is the normalized mean which is in the range $[0,1]$.

The normalized mean specifies the threshold at which the Euclidian distance is specified. The hue angle and saturation are affected by the light conditions at which the image is taken. Therefore, the threshold is calculated as:

thresh $=e^{- \text {Nmean }}$

The reference color and the unknown color are represented by two vectors by using the hue and saturation of these two colors as shown in figure 2 . The Euclidian distance between the two vectors is then calculated by the following equation:

$d=\left(\left(S_{2} \cos H_{2}-S_{1} \cos H_{1}\right)^{2}+\left(S_{2} \sin H_{2}-S_{1} \sin H_{1}\right)^{2}\right)^{1 / 2}$

The pixel is considered as to be the object pixel if the Euclidian distance is less than or equal to the threshold; otherwise it is considered as background. The main idea here is to develop a dynamic 
threshold which is related to the brightness of the image. When the brightness of the image is high, the threshold is small, and vice versa. This will allow the luminance image to control the relation between the reference pixel, and the unknown pixel.

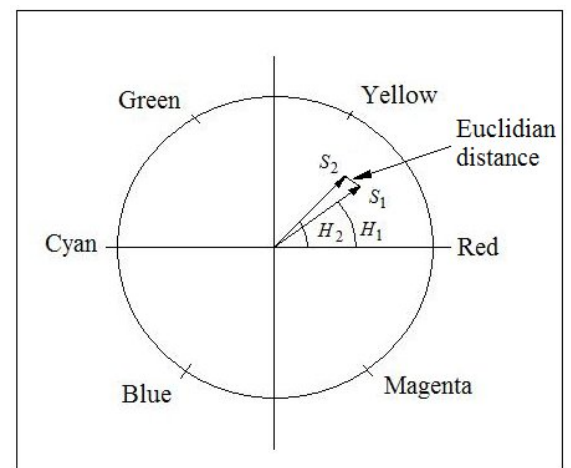

Figure 2 The Vector Model of the Hue and Saturation.

\subsection{Method 2}

This method is based on the segmentation by region growing. First, the RGB image is converted into IHLS color space. Then, the hue image is segmented according to color of the sign under consideration. Normally, a range of hue angles is specified where the color of the sign can be found. The output of this step is a binary image for the probable candidates.

This binary image is also used to calculate the seeds for the saturation image. It is divided into $16 \times 16$ pixels sub-regions, and a seed is set at the center of each sub-region if enough hue pixels are found in the binary image generated by hue segmentation. The number of sufficient hue pixels is specified by one third of the area of each subregion.

The seeds generated by the former step together with the saturation image are used as input to the region growing algorithm. The saturation image is segmented by these seeds to generate another binary image representing the candidate objects of road signs.

The final step is to apply a logical AND of the two binary images; i.e. the hue binary image, and the saturation binary image. The result is a segmented image containing the road sign with the specified color.

\subsection{Method 3}

This is a modified version of the method described by de la Escalera et al. [11]. In this method, the RGB image is converted into the IHLS color space, and both the saturation and hue are normalized to be $[0,255]$. To avoid the achromatic area defined by Vitabile et al. [10], the minimum and maximum values of the saturation are chosen to be $S_{\min }=51, S_{\max }=170$, and saturation is then calculated as follows:

$$
S_{\text {out }}= \begin{cases}0 & 0 \leq S_{\text {in }} \leq S_{\min } \\ S_{\text {in }} & S_{\min }<S_{\text {in }}<S_{\max } \\ 255 & S_{\max } \leq S_{\text {in }} \leq 255\end{cases}
$$

The hue is calculated by:

$$
H_{\text {out }}=\left\{\begin{array}{l}
255 \quad H_{\min } \leq H_{\text {in }} \leq H_{\max } \\
0 \quad \text { otherwise }
\end{array}\right.
$$

A logical AND between $S_{\text {out }}$ and $H_{\text {out }}$ will generate a binary image containing the road sign with the desired color.

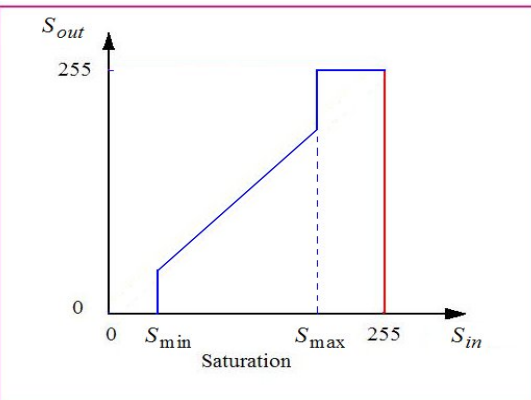

Figure 3 The Saturation Transfer Function.

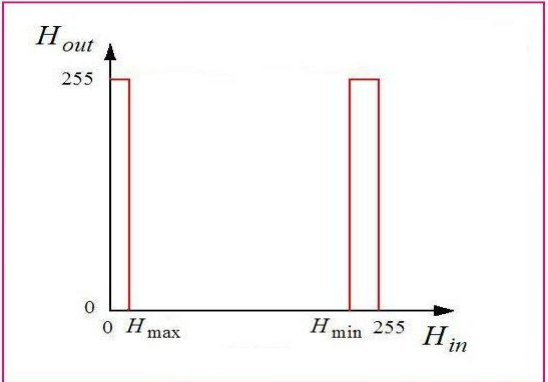

Figure 4 The Hue Transfer Function of Red.

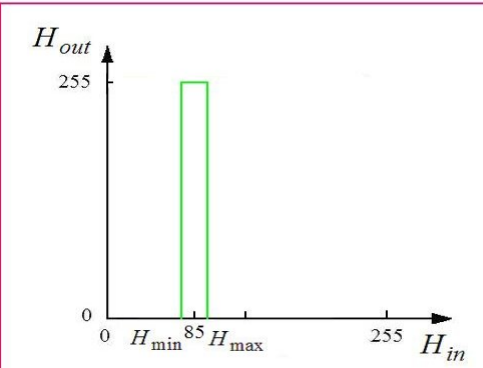

Figure 5 The Hue Transfer Function of Green.

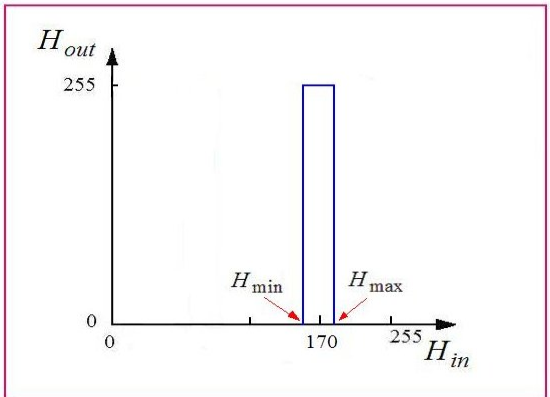

Figure 6 The Hue Transfer Function of Blue. 


\section{Results and Conclusions}

This paper shows three new methods for color segmentation used for traffic signs. The methods are based on invoking the IHLS color space, and all of them use hue and saturation to generate a binary image containing the road sign of a certain color. The IHLS color space showed very good stability to represent the hue and saturation in outdoor images taken in different light conditions. The methods are tested on more than a hundred images under different light conditions (sunny, cloudy, foggy, and snow conditions) and different backgrounds. They established very good robustness. Method 1 showed the best detection results followed by method 2, and method 3, respectively. This is due to the fact that only a single color is specified as a reference color on color circle shown in figure 2, while a range of hues and saturations are specified in methods 2 , and 3 . This allows objects with similar colors to be detected by methods 2 and 3. Some results are shown in figure 7 , where different colors are detected under different light conditions. Combining these results with shape recognition of the road signs, and pictogram recognition, which are parts of the future work, will give a good means to build a complete system which provides the drivers with the information about the signs in real time as part of the intelligent vehicle. The other key points for further study is the effect of the shadows on the stability of the hue in outdoor images, and how to deal with the reflections generated by the sign which changes the characteristics of the hue perceived by the camera. This paper is part of the sign recognition project conducted by Dalarna University / Sweden jointly with the Transportation Research Institute - Napier University / Scotland to invoke digital image processing and computer vision in the ITS field.

\section{Dedication:}

In memory of my mother and father. I will never forget you.

\section{References:}

[1] C. Fang, C. Fuh, S. Chen, and P. Yen, "A road sign recognition system based on dynamic visual model," presented at Proc. 2003 IEEE Computer Society Conf. Computer Vision and Pattern Recognition, Madison, Wisconsin, 2003.

[2] C. Fang, S. Chen, and C. Fuh, "Road-sign detection and tracking," IEEE Trans. on Vehicular Technology, vol. 52, pp. 13291341, 2003.

[3] L. Estevez, and N. Kehtarnavaz, "A real-time histographic approach to road sign recognition," presented at Proc. IEEE Southwest Symposium on Image Analysis and Interpretation, San Antonio, Texas, 1996.

[4] A. de la Escalera, L. Moreno, E. Puente, and M. Salichs, "Neural traffic sign recognition for autonomous vehicles," presented at Proc. 20th Inter. Conf. on Industrial Electronics Control and Instrumentation, Bologna, Italy, 1994.

[5] J. Miura, T. Kanda, and Y. Shirai, "An active vision system for real-time traffic sign recognition," presented at Proc. 2000 IEEE Intelligent Transportation Systems, Dearborn, MI, USA, 2000.

[6] S. Vitabile, and F. Sorbello, "Pictogram road signs detection and understanding in outdoor scenes," presented at Proc. Conf. Enhanced and Synthetic Vision, Orlando, Florida, 1998.

[7] P. Paclik, J. Novovicova, P. Pudil, and P. Somol, "Road sign classification using Laplace kernel classifier," Pattern Recognition Letters, vol. 21, pp. 1165-1173, 2000.

[8] S. Vitabile, G. Pollaccia, G. Pilato, and E. Sorbello, "Road sign Recognition using a dynamic pixel aggregation technique in the HSV color space," presented at Proc. 11th Inter. Conf. Image Analysis and Processing, Palermo, Italy, 2001.

[9] S. Vitabile, A. Gentile, G. Dammone, and F. Sorbello, "Multi-layer perceptron mapping on a SIMD architecture," presented at Proc. the 2002 IEEE Signal Processing Society Workshop, 2002.

[10] S. Vitabile, A. Gentile, and F. Sorbello, "A neural network based automatic road sign recognizer," presented at Proc. The 2002 Inter. Joint Conf. on Neural Networks, Honolulu, HI, USA, 2002.

[11] A. de la Escalera, J. Armingol, and M. Mata, "Traffic sign recognition and analysis for intelligent vehicles," Image and Vision Comput., vol. 21, pp. 247-258, 2003.

[12] G. Jiang, and T. Choi, "Robust detection of landmarks in color image based on fuzzy set theory," presented at Proc. Fourth Inter. Conf. on Signal Processing, Beijing, China, 1998.

[13] N. Hoose, Computer Image Processing in Traffic Engineering: John Wiley \& sons Inc., 1991.

[14] P. Parodi, and G. Piccioli, "A feature-based recognition scheme for traffic scenes," presented at Proc. Intelligent Vehicles '95 Symposium, Detroit, USA, 1995.

[15] J. Plane, Traffic Engineering Handbook: Prentice Hall, 1992.

[16] M. Lalonde, and Y. Li, "Road sign recognition. Technical report, Center de recherche informatique de Montrèal, Survey 
of the state of Art for sub-Project 2.4, CRIM/IIT," 1995.

[17] M. Blancard, "Road Sign Recognition: A study of Vision-based Decision Making for Road Environment Recognition," in Visionbased Vehicle Guidance, I. Masaki, Ed.

Berlin, Germany: Springer-Verlag, 1992, pp. 162-172.

[18] S. Buluswar, and B. Draper, "Color recognition in outdoor images," presented at Proc. Inter. Conf. Computer vision, Bombay, India, 1998.

[19] P. Paclik, and J. Novovicova, "Road sign classification without color information," presented at Proc. Sixth Annual Conf. of the
Advanced School for Computing and Imaging, Lommel, Belgium, 2000.

[20] A. Hanbury, and J. Serra, "A 3D-polar coordinate colour representation suitable for image analysis," Computer Vision and Image Understanding, 2002.

[21] J. Angulo, and J. Serra, "Color segmentation by ordered mergings," presented at Proc. Int. Conf. on Image Processing, Barcelona, Spain, 2003.

[22] S. Buluswar, and B. Draper, "Nonparametric classification of pixels under varying outdoor illumination," presented at ARPA Image Understanding Workshop, 1994.
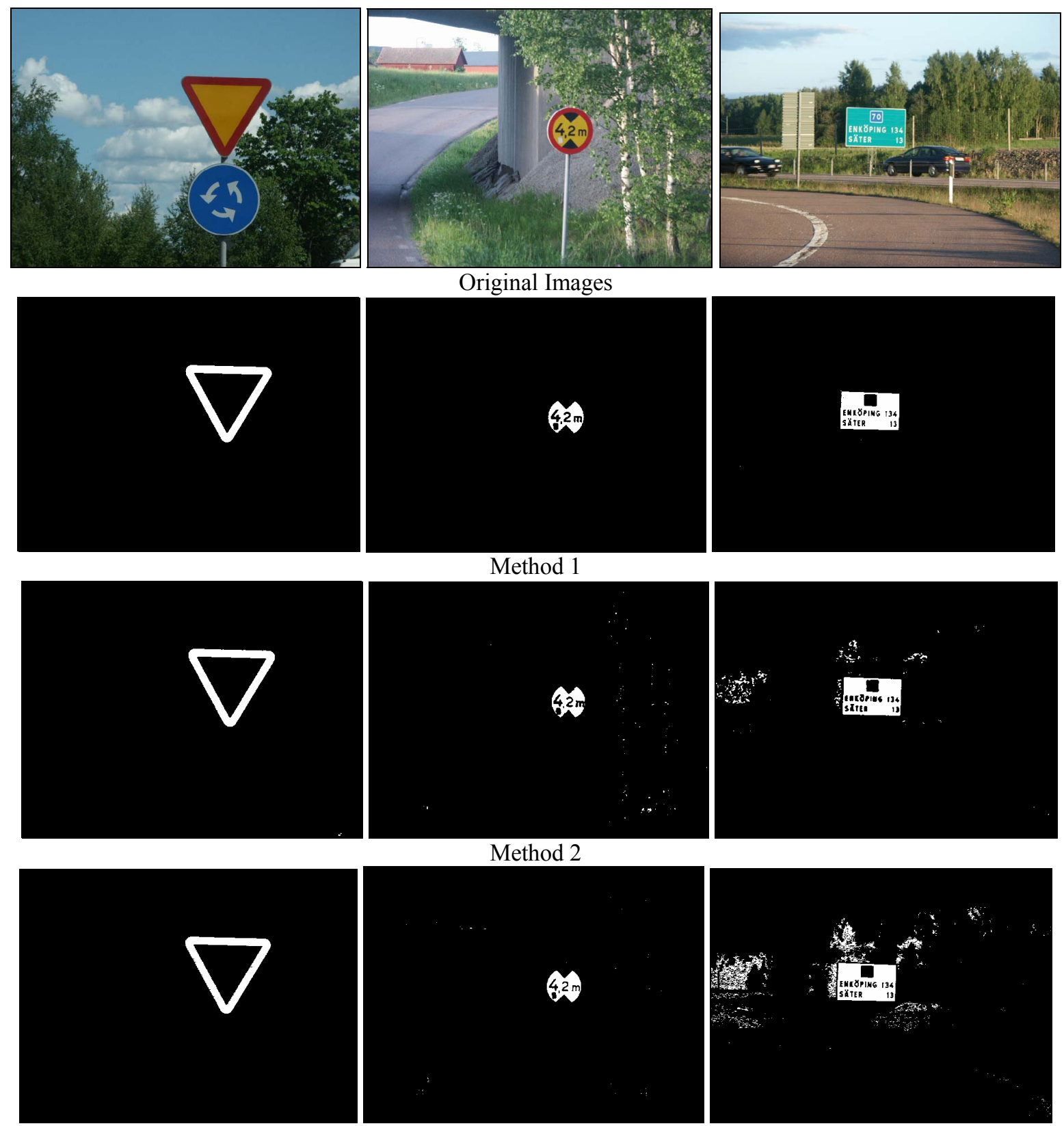

Original Images
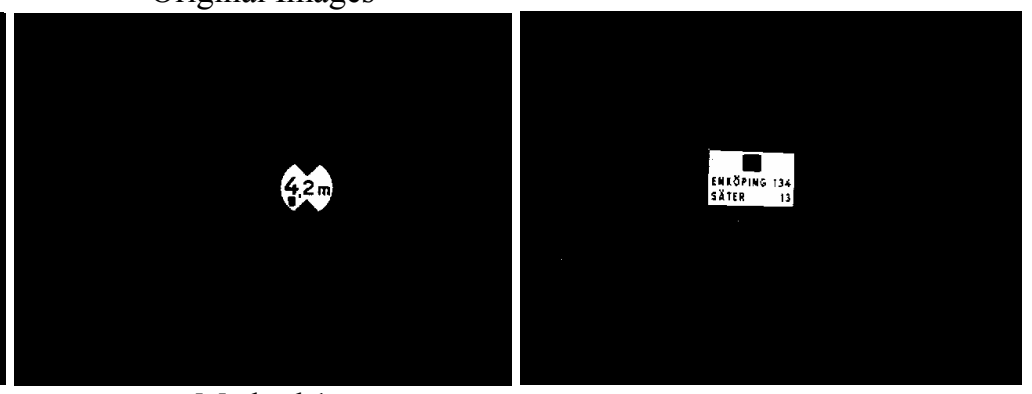

Method 1
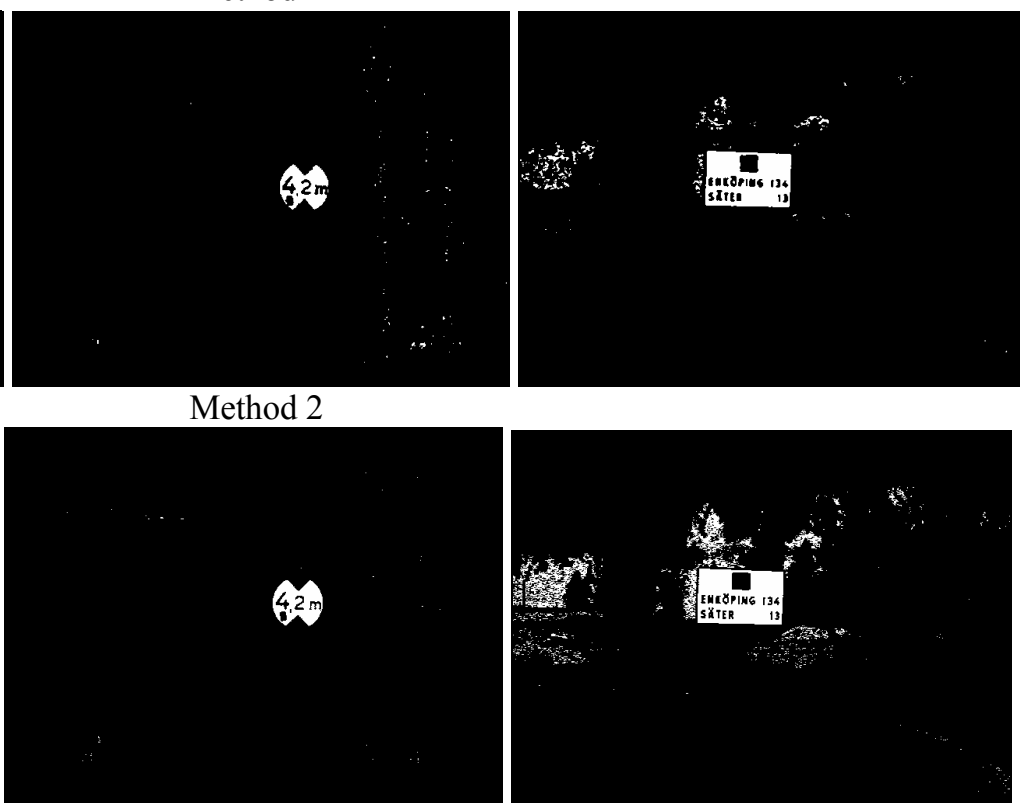

Method 3

Figure 7 Results of applying Color Segmentation Methods. 